\title{
Sprachabenteuer: Yoko Tawadas exophone Erkundungen des Deutschen
}

\author{
MARKO PAJEVIĆ
}

\begin{abstract}
Adventures in Language: Yoko Tawada's Exophonic Explorations of German. Yoko Tawada (1960) is for good reason one of the prime examples for contemporary German exophonic literature. She is a very successful writer in Japanese and in German and provides in her Germanophone writings an ethnography of the German worldview, as Wilhelm von Humboldt famously called languages, or of the German language-mindset. This article focuses on her 2010 poetry volume Abenteuer der deutschen Grammatik ('Adventures of German Grammar') to demonstrate how exophonia can allow us to develop an acute awareness of the ways in which language structures shape our patterns of thinking. Coming from a very differently organised language, Japanese, Tawada comments in playful ways on the implications of German, and compares it translinguistically with Japanese. Looking at German from an outside position enables her to be very creative and to make Germans discover their language with new eyes. Translingual writing, even though also present in a real mixing of languages in Tawada, appears here as a way to understand how much our ideas are shaped by our linguistic structures, and that there are alternative worldviews. It thus contributes greatly to a relativisation of one's own perspective and helps to open up to difference and creativity.
\end{abstract}

Keywords: Yoko Tawada; exophonic writing; translingualism; worldview; multilingualism; adventure

\section{Abenteuer und Sprache}

Abenteuer sind spannend: sie holen uns aus unserer alltäglichen Routine heraus und geben uns das Gefühl intensiv gelebten Lebens. Sie sind Herausforderungen, die uns an unsere Grenzen bringen und uns die befriedigende Erfahrung erlauben, mutig unabgestecktes Gebiet zu betreten, die Grenzen unserer Welt zu weiten, und derart, indem wir das Unbekannte meistern, wachsen wir über uns hinaus und entwickeln unsere Persönlichkeit. Es gibt einen ganzen Bereich der Pädagogik, der auf diesem Gedanken aufbaut, dass Abenteuer die Fähigkeit zu einem selbstbestimmten Leben herausbilden. Das hat dann Begriffe hervorgebracht wie Abenteuerspielplatz, 
PAJEVIĆ

Abenteuertourismus und sogar Abenteuerautobahnraststätte. Heutzutage, vor allem im Deutschen, wird der Ausdruck Abenteuer häufig für eine kurzzeitige Liebesgeschichte eingesetzt, ein Liebesabenteuer - der Begriff vermittelt einen Hauch von Frivolität, zumindest einen Mangel an Ernsthaftigkeit. Ursprünglich jedoch stammt eine aventiure aus der französischen Ritterkultur und bezeichnete ein ernsthaftes Unternehmen von kultureller Bedeutung, für welches Risiken eingegangen werden. Im 13. Jahrhundert bezeichnete es auch ein Wunder. Ein Abenteuer - das Wort führt das mit sich - ist etwas, was uns widerfährt, ein Advent, also die Ankunft eines Ereignisses. Wir können es suchen, aber es ist immer das Abenteuer, das uns findet. Wir haben keine Kontrolle darüber. Es ist unbekannt und unvorhersehbar.

All dies wird nicht unmittelbar an Grammatik denken lassen, zumindest die meisten Leute nicht. Yoko Tawada jedoch hat Abenteuer der deutschen Grammatik geschrieben, so der Titel eines Zyklus' von 20 Gedichten (Tawada 2010). Tawada ist eine sowohl in Deutschland als auch in Japan hochangesehene Schriftstellerin, die in beiden Sprachen schreibt. 1960 geboren, kam sie 1982 nach Hamburg, um dort zunächst zu arbeiten und später deutsche Literatur zu studieren. Ihre Promotion wurde von Sigrid Weigel betreut, mit der sie eine enge Freundschaft verbindet. Sie veröffentlichte 1987 ihr erstes Buch auf Deutsch, allerdings noch in Übersetzung. Seither hat sie weit über 1000 Lesungen in der ganzen Welt gegeben, Dutzende von Büchern veröffentlicht - Romane, Dramen, Gedichte und, meines Erachtens am Interessantesten: Essays - und viele Preise und Ehrungen in beiden Ländern erhalten. 2006 zog sie von Hamburg nach Berlin, wo sie bis heute lebt, allerdings verbringt sie auch viel Zeit in den USA, wo sie sowohl von Germanisten als auch von Japanologen hofiert wird. Ihr Werk wird viel studiert als herausragendes Beispiel transnationaler und exophoner Literatur, also Literatur, die in einer Zweitsprache geschrieben wurde und in der Spuren der ersten Sprache in irgendeiner Form vorkommen und thematisiert werden. Ihre Schriften stellen eine Spielart der Ethnographie dar, die deutsche Sprache und Kultur von außen betrachtet und durch diesen ungewöhnlichen Blickwinkel erhellende Einblicke in das bietet, was von Innen $\mathrm{zu}$ offensichtlich ist, um gesehen zu werden. Sie erlaubt uns, unsere Welt neu zu entdecken, die „Zeichen der Welt neu lesen zu lernen“, wie Jürgen Wertheimer formulierte (im Nachwort zu Tawada 2001: 61). Die meisten dieser Beobachtungen spielen sich innerhalb des Sprachlichen ab, was verdeutlicht, dass und wie wir „aus Sprachzeichen Wirklichkeitskörper formen“ (Wertheimer in Tawada 2001: 62). Sie beschreibt etwa ihre Verlegenheit, als ihr bewusst wurde, dass ein Bleistift im Deutschen männlich ist - im Japanischen gibt es keine Geschlechter - diese Sexualisierung der Welt war für sie mit Scham verbunden (Tawada 2011: 9-12). 


\section{Tawadas mehrsprachiges Denken}

Ein Kind, oder eine Person, die eine Fremdsprache spricht, nimmt die Sprache beim Wort (vgl. Tawada 2011: 13). Was auch bedeutet, dass die Welt in der Sprache erschaffen wird. Das führt zu der Frage, ob ich eine andere Person werde, wenn ich eine andere Sprache spreche. Oder ob ich die Welt in einer anderen Sprache anders sehe? Sieht zum Beispiel ein Seepferdchen im Deutschen anders aus als ein tatsu-no-otoshigo? - Das ist das japanische Wort für Seepferdchen und bedeutet wortwörtlich „das verlorene Kind des Drachens" (Tawada 2016: 29).

Es ist offensichtlich kein Zufall, dass eine Nicht-Muttersprachlerin den Gedanken von Abenteuern der deutschen Grammatik hervorbringt. Unsere erste Sprache ist natürlich auch unsere erste Fremdsprache, wie Tawada betont. Wir müssen sie als kleine Kinder lernen und dann verwundert sie uns wohl mindestens ebenso sehr wie jedwede andere Sprache, die wir später lernen. Sobald wir diese Erstsprache jedoch gelernt haben, stellt sie unser Denkuniversum dar. Sie wird die natürliche Perspektive auf die Welt solange, bis wir mit alternativen Weisen die Welt zu sehen konfrontiert werden, das hei $\beta t$, mit anderen Sprachen, die wir lernen. Eine neue Sprache lehrt uns, dass unsere Vorstellungen von der Welt nicht die einzig möglichen sind, dass wir andere Perspektiven einnehmen können - auf die Dinge, auf die Beziehungen zwischen den Dingen, oder zwischen uns und den Dingen bzw. uns und anderen, das heißt auf Beziehungen allgemein. Sprache ist tatsächlich das Medium all unserer sozialen Beziehungen, einschließlich derjenigen zu uns selbst (vgl. Joseph 2019: 16). Insofern ist es Sprache, die unser Leben bestimmt.

Tawada geht so weit zu behaupten, dass Sprache in ihrer physischen Vibration unser Fleisch durchläuft und die sprechende Person körperlich transformiert, was erklärt, warum die Gesichter von Emigranten in den Nachfolgegenerationen anders aussehen als die der im Vaterland verbliebenen - unsere Gesichtszüge sind nicht nur durch Genetik bestimmt, sie werden dadurch geformt, was die Sprache mit uns macht (vgl. Tawada 2001: 8). ${ }^{1}$ Tawada formuliert sogar, dass es die Sprache ist, die die Menschen schreibt, nicht umgekehrt (vgl. Heimböckel in Gutjahr 2012: 157). Die Sprache prägt sich demzufolge also ganz konkret dem Geist und dem Körper der Sprechenden ein. Die Bedeutung des Klangkörpers der Sprache ist ein wiederkehrendes Thema: Tawada betont die grammatische Relevanz des Tonfalls und auch die herausragende Bedeutung von Klang und Intonation für Kinder

Vgl. auch Talisman, p. 52: „das Gesprochene gelangt durch das Gehirn ins Fleisch“. 
PAJEVIĆ

beim Spracherwerb (vgl. Tawada 2001: 8-9), ${ }^{2}$ ebenso wie die musikalische Dimension für Erwachsene, die eine neue Sprache erlernen. Wenn sie Deutsch spricht, stellt Tawada fest, fühlt sie sich wie ein Komponist, der den Klang von Waldvögeln hört, schreibt und nachahmt (Tawada 2001: 22). ${ }^{3}$

Der Körper der Sprache, aber auch der eigene Körper findet in seine Kraft in einer Fremdsprache, wir erhalten ,Körperbewusstsein' (vgl. Tawada/ Gutjahr 2012: 28-29. Sie lenkt unsere Aufmerksamkeit auf die viel klareren Umrisse und die Konkretion in Fremdsprachen, im Vergleich zu unserer Erstsprache, in welcher die Selbstverständlichkeit uns davon abhält, solche Dinge zu bemerken (vgl. Tawada 2001: 7). Das folgende Zitat verdeutlicht das noch weiter: „Die Schwierigkeiten werfen Licht auf den Sprachkörper und machen ihn auf diese Weise sichtbar. Dagegen bleibt man meistens blind in einer Sache, die man beherrscht." (vgl. Tawada 2001: 25) Dinge, mit denen wir zu vertraut sind, sehen wir nicht.

In einer neuen Sprache setzen wir uns also dem Unbekannten aus, neuen Lebensformen. Und wir werden bewusster. Das ist in der Tat ein Abenteuer. Wenn wir uns derart mit neuen Formen und Perspektiven konfrontieren oder, um es mit Wilhelm von Humboldts Ausdruck für Sprachen zu sagen: mit Weltansichten - dann gehen wir Risiken ein. Wir müssen eventuell komfortable Gewissheiten aufgeben, unsere Überzeugungen mögen erschüttert werden. Nicht jeder mag Abenteuer, wenn sie Wirklichkeit werden und Konsequenzen für unser eingerichtetes Leben zeitigen. Solche Abenteuer können allerdings auch unseren Geist öffnen und weiten. Es ist ein wenig wie Jakobs Kampf mit dem Engel (Genesis 32:22-32): indem wir uns dem Unbekannten aussetzen, werden wir beeindruckt, erhalten wir eine Prägung, und werden, derart, verwandelt. Jakob hinkte nach seinem Kampf mit dem Engel, aber er gewann auch einen neuen Namen, Israel, ein bleibender Eindruck, und schuf so die Grundlage für sein zukünftiges Volk. Er gewann Bewusstsein und Perspektive.

2 Dirk Weissmann, zum Beispiel, lenkt ebenfalls die Aufmerksamkeit auf diese semantische Dimension in der lustvollen Beschäftigung mit Klang und Gesten. Hören beinhaltet eine Offenheit gegenüber anderen semantischen Bedeutungsdimensionen (vgl. Weissmann in Dembeck/Uhrmacher 2016: 188-189).

3 In den Bühnenanweisungen zu ihrem Theaterstück Till, in dem Japaner und Deutsche auf der Bühne stehen und in ihrer jeweiligen Sprache sprechen, fordert sie ausdrücklich, dass beide Sprachen unübersetzt bleiben, selbst wenn Teile des Stückes damit für nahezu das gesamte Publikum unverständlich bleiben. Sie stellt dem jedoch musikalische oder visuelle Zugänge zum Bühnengeschehen entgegen (vgl. Tawada 1998: 43-44). Und in Tawada 2018: 150 behauptet sie, dass für sie nichts schöner sei als stundenlang in einem Theater zu sitzen und einer Sprache zuzuhören, sie sie nicht versteht. 
Tawada sieht das Element der Abstraktion und Entfremdung in diesem erweiterten Bewusstsein des Lebens in einer Fremdsprache. Sie kommentiert allerdings: „Aber ich sehe eine Chance in dieser zerstörten Beziehung zur Muttersprache und zur Sprache überhaupt. Man wird ein Wortfetischist“ (Tawada 2016: 32). Es geht um die Präzision unserer Sichtweise.

Tawada beschreibt die Unmöglichkeit, sich Leben und Denken in einer anderen Sprache vorzustellen, wenn man einsprachig ist (vgl. Tawada/ Gutjahr 2012: 19). Wenn man jedoch erst einmal einen mehrsprachigen Geist entwickelt hat, wird es unvorstellbar, ohne Mehrsprachigkeit zu leben. Sie betrachtet sich selbst jetzt als ein zweisprachiges Wesen, in dem Sinne, dass beide Sprachen gegenseitig wichtig sind und sie nicht mehr ohne die je andere Sprache sein kann, da beide einander erhellen (vgl. Tawada/Gutjahr 2012: 19). Folglich gibt es auch den Genuss in diesem Abenteuer des Wandelns auf ungewohnten Pfaden, etwas, was Tawada mit dem schönen Wort Heimatlust bezeichnet, ein Wort, welches Heimatverlust anklingen lässt und es zugleich verdreht, aber ebenso auf die Wanderlust in solchen Abenteuern anspielt, und sie definiert folgendermaßen: „Das ist die Lust, die man empfindet, wenn man die eigene Heimat mit einem neuen Blick betrachtet." (Tawada 2011: 119)

\section{Das Beispiel der Personalpronomina}

Lassen Sie uns jetzt also diese Wanderlust genießen und auf eine kleine Abenteuerreise mit Yoko Tawada gehen, einen genaueren Blick auf ihre Abenteuer mit der deutschen Grammatik werfen, um dann einige Schlussfolgerungen dafür zu ziehen, was das für Mehrsprachigkeit bedeutet, für uns selbst, sogar für das Menschsein, denn, wenn die Sprache das menschliche Dasein bestimmt, dann gibt es auch immer eine anthropologische Dimension in dem, was im Sprachlichen geschieht. Leibniz hat Sprachen bereits „le meilleur miroir de l'esprit humain“" genannt (Leibniz 1966: 290), den besten Spiegel des menschlichen Geistes: sie verdeutlichen, was es heißt, ein Mensch zu sein. Und Leibniz betonte ebenfalls die Bedeutung der Mehrsprachigkeit für unseren Geist, indem er „la merveilleuse variété de ses opérations“, also die wunderbare Vielfalt seiner Operationen, als wünschenswert pries (Leibniz 1966: 239). Tawada bestätigt das: „Das wichtigste Erbe der Menschheit besteht jedoch aus den Sprachen mit ihrer Unterschiedlichkeit. Warum sollte man sie nicht mit anderen teilen? Durch das Teilen wird das Erbe nicht weniger, sondern mehr." (Tawada 2016: 21) 
PAJEVIĆ

Der Gedichtzyklus Abenteuer der deutschen Grammatik ist palimpsestartig auf dem Hintergrund eines deutschen Grammatikbuches gedruckt. ${ }^{4}$ Allein diese Aufmachung verdeutlicht die Überlappungen von Grammatik und Dichtung und suggeriert, dass wir uns auf fremdem Boden bewegen, wo Begegnungen und unerwartete Verbindungen stattfinden können. Tawada hebt eine Reihe von Besonderheiten der deutschen Grammatik hervor, die für einen deutschen Muttersprachler ganz natürlich scheinen und normalerweise unhinterfragt bleiben, etwa die Großschreibung gewisser Worte (7), Nominalisierungen (10), Aussprache (12), Zeichensetzung (13), Anglizismen (14) und Wortstellung (18). Ich werde mich im Folgenden auf die Personalpronomen am Beispiel dreier Gedichte konzentrieren.

Tawada spricht in ihrem Werk immer wieder über ihre Beziehung zum deutschen Wort Ich. Sie behauptet, ihr japanisches Ich unterscheide sich vom deutschen. Der Grund dafür liegt in unterschiedlichen Traditionen der Subjektivität und von Sinn. Im Haiku etwa gibt es kein beobachtendes Ich, keine Dualität zwischen Sehen und Gesehen-Werden, zwischen Subjekt und Objekt. Ein Haiku stellt ausschließlich einen Moment dar, ohne beobachtende Person. Im Japanischen gibt es viele Wörter für Ich, jedes verweist auf eine andere Identität, einen anderen Status oder ein anderes Geschlecht. Im Deutschen ist Ich einfach Ich, es bezieht sich ausschließlich auf die sprechende Person, unabhängig von allen Eigenschaften dieser Person - weshalb es Tawadas Lieblingswort ist (vgl. Tawada 2018: 55 und 59). Wenn sie das deutsche „Ich“ verwendet, bedeutet dies für sie keine soziale Rolle oder Position, es stellt für sie eine Linse dar, welche ihr das Sehen ermöglicht. Aus diesem Grund betrachtet sie das Ich auch nicht als schwierig, wie Deutsche es wohl tun mögen, für sie ist es nicht mit Geschichte und Verantwortung aufgeladen. Sie rät dazu, das Ich rein grammatikalisch zu betrachten, dann wäre es befreiend (vgl. Tawada/Gutjahr 2012: 26-27).

Das ist, was sie im folgenden Gedicht macht.

Die zweite Person Ich

Als ich dich noch siezte, sagte ich ich und meinte damit mich.

\footnotetext{
4 Monika Schmitz-Emans analysiert Tawadas Einsatz der Schrift und wie diese Präsentation die Lesenden sensibilisiert: „Die Sensibilität für Gestalt und Faktur von Geschriebenem wird vom Befremden angesichts eines unvertrauten Schriftbildes stimuliert" (Schmitz-Emans in Gutjahr 2012: 284).
} 
Sprachabenteuer: Yoko Tawadas exophone Erkundungen des Deutschen

Seit gestern duze ich dich,

weiß aber noch nicht, wie ich mich umbenennen soll.

(Abenteuer der deutschen Grammatik, 8 @Konkursbuch Verlag Claudia Gehrke)

Der Titel des Gedichts ist bereits eine Provokation, indem es die erste Person Ich als zweite bezeichnet. Das Ich erhält hiermit den Status eines Anderen, jemand, der von außen betrachtet werden kann, vom Selbst losgelöst oder ganz ohne diese Instanz des Selbst. Das führt auch den Umstand mit sich, dass es mehr als ein Ich gibt, was die traditionelle westliche Vorstellung vom determinierten selbstbestimmten Subjekt von sich weist.

Das Gedicht thematisiert den Unterschied zwischen dem formellen Siezen und dem informellen Duzen. Das lyrische Ich gesteht seine oder ihre Verwirrung bezüglich des Status und der Bezeichnung des Ich, wenn es die Beziehung zu einem Du verändert. Das das Du formell ansprechende Ich muss, so das Gedicht, anders sein als das das Du informell ansprechende Ich. Jedoch ändert sich nichts am Wort Ich. Dieser Umstand ruft tatsächlich die Frage nach unserer Konzeption des Ich auf den Plan - existiert es überhaupt auch für sich allein oder ist es nicht eher immer durch seine Beziehungen bestimmt? Sind wir einfach, oder können wir nur in Beziehung zu einem Anderen sein, dialogisch?

Solche Fragen verdeutlichen die Relevanz grammatischer Figuren für unsere Weltansicht und, folglich, für die grundlegendsten philosophischen Begriffe. Barbara Cassins philosophisches Wörterbuch der Intraduisibles, der Unübersetzbarkeiten, hat viel zur Bewusstwerdung beigetragen, dass Begriffe aus Wörtern bestehen und dass diese unterschiedlich in verschiedenen Sprachen sind, was bedeutet, dass auch die Begriffe nicht dieselben sind, selbst wenn sie direkte Übersetzungen zu sein scheinen (Cassin 2004). Das bedeutet wiederum, dass in verschiedenen Sprachen tatsächlich anders gedacht wird, dass sie andere Vorstellungen von der Welt transportieren bzw. schaffen. Sprachen sind nicht einfach Werkzeuge für die Kommunikation, sie sind in erster Linie Kognition. Das basiert sehr stark auf Humboldts Begriff der Weltansicht. Tawadas Gedicht macht diesen in den Sprachstrukturen inhärenten Unterschied im Denken evident, den deutschen Lesern wird das Deutschsein ihres Denkens unter die Nase gerieben und derart treten sie aus dem festen Rahmen ihrer Weltansicht hinaus und sie werden befähigt, außerhalb ihrer gewohnten Pfade zu denken. Beide Seiten unternehmen ein Abenteuer: die japanische Schriftstellerin, die versucht, die Konzeption des ,Ich' im Deutschen zu begreifen, und die deutschen Leser, die ihre Vorstellungen vom Ich erweitern. Grammatik ist hier eine Möglichkeit, neue Denkräume zu betreten - das ist riskant, weil es alte Überzeugungen destabilisiert, aber auch aufregend, weil es neue Perspektiven eröffnet. 
PAJEVIĆ

Das wird noch weiter verkompliziert durch den Umstand, dass das „ich“ das „mich“ und das „dich“ durchzieht (vgl. Ette in Gutjahr 2012: 328). Ich, dich und mich sind verbunden durch die Sprachform. Sowohl mich und dich enthalten das ich: man kann dich und mich nicht ohne das Ich denken.

Tawada kommentiert, dass das Ich dieses Gedicht nichts mit ihr selbst zu tun habe. Es sei inszeniert, um eine Kohärenz zu schaffen, aber ,natürlich ' ist es nicht kohärent, da es Mehrfaches bedeuten kann (Tawada/Gutjahr 2012: 27).

Das folgende Gedicht baut diese Frage nach den Personalpronomen und nach der Identität aus:

Die zweite Person

Du hast ein Geschlecht.

„Du“ hat kein Genus.

Du da!

Meinst du mich?

Ja!

Dann ist dein „Du“ heute weiblich.

„Ich“ hat kein Genus.

Und das ist ein Genuss für mich.

„Ich“ sagt mein Freund, der einen Freund hat.

Er ist ein Ich, wenn sein Mund sich bewegt.

Er ist ein Du, wenn seine Ohren mir zuhören.

Egal ob dich eine Sie oder ein Er lieben, immer bist du eine zweite Person und geschlechtslos.

(Abenteuer der deutschen Grammatik, 23 CKonkursbuch Verlag Claudia Gehrke)

Dieses Gedicht legt den Schwerpunkt auf die zweite Person, das Du, aber es hat auch Implikationen für das Ich, selbstverständlich. Das Gedicht lenkt unsere Aufmerksamkeit auf den Umstand, dass eine Person ein Geschlecht hat, aber das Pronomen ,Du' hat keines. Das Gleiche gilt für das Ich. Die gleiche Person ist ein Ich oder ein Du, je nachdem ob man spricht oder zuhört. Das erste und das zweite Personalpronomen im Deutschen sind vorurteilslos in Bezug auf Homo- oder Heterosexualität. Wenn wir von einem Ich und einem Du und deren Beziehung sprechen, hat Geschlecht dabei keine Bedeutung, wenigstens grammatikalisch.

Das nächste Gedicht gibt einen Einblick in die dritte Person: 
Sprachabenteuer: Yoko Tawadas exophone Erkundungen des Deutschen

Die dritte Person

Er trägt seinen alten Hosenträger, sie ihren weißen Busenhalter.

Ein Ich hingegen läuft nackt herum.

Ein Ich kann Marie, Marika oder Mario heißen.

Er erträgt seine unerträgliche Mutter, sie ihren Vater.

Sie trägt eine Gebärmutter in sich, er seine Hoden.

DU trägst nichts bei dir außer den Buchstaben D und U.

Ein Du kann Kain, Cathy oder Keika heißen.

„Ich“ muss keine Steuern zahlen, denn ICH ist kein bürgerlicher Name.

„Du“ musst nicht zur Bundeswehr. Ein Soldat, der DU heißt, tötet nicht.

(Abenteuer der deutschen Grammatik, 24 CKonkursbuch Verlag Claudia Gehrke)

Dieses Gedicht weist darauf hin, dass die dritte Person, im Gegensatz zur ersten und zweiten Person, gegendert ist. Im Deutschen, anders als im Französischen etwa, bezieht sich das Possessivpronomen auf das Subjekt, nicht auf das Objekt. Die Pronomen der ersten und zweiten Person können von allen gebraucht werden - solange sie nicht als dritte Person betrachtet werden, sind sie nicht zum Objekt gemacht. Das bedeutet, sie können nicht biopolitisch betrachtet werden. Für den Staat und die Institutionen gibt es keine Ichs oder Dus. Die letzte Zeile betont auch die dialogische Dimension der ersten beiden Personen, ganz im Sinne von Martin Bubers dialogischem Prinzip (Buber 1962): es ist unmöglich, in einer Ich-Du-Beziehung zu töten, die Voraussetzung für Krieg ist ein objektivierter ,Feind' in der dritten Person.

Das waren nur einige wenige Beispiele, wie Grammatik eine Erkundung des Lebens, ein Abenteuer des Geistes sein kann. Um uns unserer Denkmuster und -begrenzungen - bewusst zu werden und sie derart zu überwinden, ist eine Außenperspektive sehr hilfreich. Eine neue Sprache ist deshalb eine Denkschule. Eine neue Sprache zu lernen ermöglicht es uns, auf neue Weise zu denken, aber auch um das Alte zu denken, und es anders zu denken, es zu überdenken. Eine andere Weltansicht erlaubt mehr Freiheiten, wie Tawada explizit sagt (Tawada/Gutjahr 2012: 35). 
PAJEVIĆ

\section{Die Konsequenzen für ein mehrsprachiges Denken}

Das daraus folgende Bewusstsein von anderen Denkweisen und von der Vielfalt der Möglichkeiten kann in der Tat befreiend wirken, wenn die betreffende Person Differenz erträgt und nicht angst- oder hasserfüllt ist - in dem Falle allerdings mag das Andere als Bedrohung empfunden werden und müsste dann bekämpft werden. Aber nichtsdestotrotz, ein Bewusstsein, dass unterschiedliche Weltansichten existieren, sollte eher zu mehr Toleranz führen und zu dem Bedürfnis, Sichtweisen auszutauschen, um zu wachsen und den menschlichen Geist in seiner Fülle zu verstehen. Wir sprechen hier weniger über Mehrsprachigkeit als über die Fähigkeit, eine andere Sprache zu bewohnen, eine andere Weltansicht zu leben. Mit einer solchen Haltung deckt die Vielfalt die Unendlichkeit des Lebens auf und bietet die Möglichkeit, anders zu denken, neue Perspektiven einzunehmen.

Tawada entwickelt eine Antwort auf ihre Frage, ob wir eine andere Person in einer anderen Sprache sind. Sie zieht es vor zu sagen, dass wir uns eher als ein Netz betrachten sollten, dessen Struktur immer dichter wird, indem wir neue Elemente aufnehmen und derart neue Muster schaffen, mit mehr Knoten und Unregelmäßigkeiten. Mehrsprachigkeit ermöglicht dann, mit diesem Netz den kleinsten Plankton zu fischen (vgl. Tawada 2016: 30).

Yoko Tawadas exophone Literatur ist eine Ethnographie, die es den deutschen Lesern und Leserinnen ermöglicht, ihre eigene Sprache als Fremdsprache zu erfahren, indem sie eine Außenperspektive einnehmen. Das ist die klassische Situation der Selbstreflektion und damit die Definition des Denkens und des Bewusstseins selbst. Eine Sprache ist immer eine Theorie, das heißt, wie die griechische Wurzel des Wortes impliziert, eine Weise, die Dinge zu sehen, eine Weltansicht. Max Horkheimer unterschied zwischen traditioneller Theorie, die über etwas außerhalb ihrer selbst nachdenkt, und kritischer Theorie, die sich ihrer eigenen kulturellen und historischen Determiniertheit bewusst ist und diese mit einbezieht (Horkheimer 1992), sich also ihrer eigenen Historizität bewusst ist, wie Henri Meschonnic das nennt. ${ }^{5}$ Tawadas Texte könnten

5 Historizität ist ein wichtiger Begriff in der Theorie Henri Meschonnics, im Glossar zu The Henri Meschonnic Reader definiere ich ihn folgendermaßen: „Historicity: This key term means the situatedness and subjectivity of any discourse, implying also that the critique itself is situated and part of the discourse. In Meschonnic's theory of language, this term captures the contradiction that an activity is situated and circumstantial, but can constantly leave this situation and remain active in the present, in new presences. It is opposed to historicism, which is the completed, the past of meaning and a reduction of meaning to its conditions of production. Historicity designates a relation, 
Sprachabenteuer: Yoko Tawadas exophone Erkundungen des Deutschen

deshalb kritische Texte in diesem Sinn genannt werden, da sie ein Bewusstsein ihrer eigenen Voraussetzungen darstellen. Sie spielen auf selbstreflektive und bewusste Weise mit den den Sprachen eingeschriebenen Voraussetzungen und Vorannahmen.

Exophone Literatur wie diejenige Tawadas zeigt, was eine mehrsprachige Geisteshaltung für unsere Weltansicht bewirkt. In ihrer bahnbrechenden Studie Beyond the Mother Tongue. The Postmonolingual Condition behauptet Yasemin Yildiz, dass das Paradigma der Einsprachigkeit als Norm aus einer relativ jungen Entwicklung resultiert, die am Anfang des 18. Jahrhunderts einsetzte. Dieser Zustand sei in unserer Zeit im Umbruch begriffen, so Yildiz, sodass sie in diesem Rahmen ihre These der postmonolingual condition als Kampfbegriff gegen das immer noch dominante Paradigma der Einsprachigkeit aufstellen kann (Yildiz 2012: 3-4). Till Dembeck und Anne Uhrmacher stellen aus linguistischer und literarischer Perspektive sogar die Existenz der Einsprachigkeit schlechthin in Frage und stellen fest, dass es nur Mehrsprachigkeit gibt, lediglich mehr oder weniger sichtbar (Dembeck/ Uhrmacher 2016: 9).

In ihrem Kapitel zu Tawada (Yildiz 2012: 109-142) verweist Yildiz auf die besondere Situation eines „politically charged, reemergent multilingualism“ (Yildiz 2012: 115) in Japan, gegen die ideologische Aufzwingung des Tokioter Dialekts als einziger Sprache des japanischen Nationalstaats zu Beginn des frühen 19. Jahrhunderts. Selbst wenn Tawada nicht offen politisch sein mag, so nehmen ihre Schriften an solchen gesellschaftlichen Verschiebungen teil. Yildiz sieht ebenfalls Tawadas Entscheidung, Japan zu verlassen, als repräsentativ für viele japanische Frauen an, die in den 1980ern und 1990ern das Gleiche getan haben, „yearning for the foreign“, um ihren Lebensbedingungen als Frau in Japan zu entkommen (Yildiz 2012: 122). Aus diesem Grunde betrachte Tawada ,the move to a new language environment, undertaken individually and voluntarily, as liberating and enabling" (Yildiz 2012: 120). Yildiz präsentiert Tawada als ein Beispiel für die Weise, wie Mehrsprachigkeit „affects the monolingual paradigm from within. Through homophones and homonyms, Tawada points to structures that look alike but are not, and that look like one

the indefinitely renewed encounter between past and present moments of meaning. As such, it is unpredictable. This is a central term for poetics, since it creates the specificity of a literary work and shows that the sense of literature is not limited to the sense of the words at the time of their production. This implies the necessity of a theory of discourse for all disciplines of meaning because it shows the interaction between the theory of language and the theories of literature, art, the subject, ethics and politics. This is why Meschonnic's theory of rhythm is a historical anthropology of language." (Pajević 2019) 
PAJEVIĆ

word but are not. Multiplicity and multilingualism are thus not necessarily visible, yet they constitute our world" (Yildiz 2012: 141).

Yildiz' Vorstellung von Schreiben jenseits der Muttersprache bedeutet „writing beyond the concept of the mother tongue.“ (Yildiz 2012: 14) Ihre Erklärung für das Aufkommen des Einsprachigkeitsparadigmas und ihre Schuldzuweisung an die deutschen Romantiker und ihre neu entwickelte Sprachvorstellung scheint mir jedoch problematisch $\mathrm{zu}$ sein (vgl. Yildiz 2012: 6-10). Die Etablierung der Einsprachigkeit als Norm hat einen gänzlich anderen Hintergrund: Es ist ein biopolitisches Interesse (in Michel Foucaults Vorstellung des Begriffs), welches zur gleichen Zeit aufkam. Der Staat erzwang Einsprachigkeit mit dem Ziel einer effizienteren Verwaltung seiner Untertanen und einer einheitlichen Nation. Dementsprechend gab es auch in Japan zu jener Zeit vergleichbare Tendenzen, die wohl damals nicht durch die deutschen Romantiker angeregt wurden. Yildiz hingegen nennt Herder, Wilhelm von Humboldt und Schleiermacher als Hauptverantwortliche für das Einsprachigkeitsparadigma, aufgrund von deren Vorstellung der Bedeutung der Sprache für das Denken, wodurch die prägende Kraft der Muttersprache betont wurde. Allerdings gesteht Yildiz diesen Denkern zu, dass ihre Sprachkonzeption das Studium anderer Sprachen gefördert hat und zu einer größeren Anerkennung der Sprachenvielfalt geführt habe (Yildiz 2012: 6-7). Die weitverbreitete Kritik an dieser relativistischen Sprachauffassung, die Yildiz hier aufgreift und weiterführt, vor allem, dass diese zu der Vorstellung des voneinander Abgetrenntseins der Sprechenden verschiedener Sprachen führe, ist in der Tat ein „extreme end“ (Yildiz 2012: 8), eine drastische Schlussfolgerung, die von keinem der genannten Denker in dieser Form gezogen wurde. Sie sollten nicht für extremistische Positionen verantwortlich gemacht werden.

Sie haben, ganz im Gegenteil, eine Sprachauffassung entwickelt, die es ermöglicht, den Wert von Mehrsprachigkeit und von exophoner Literatur zu würdigen, als eine Bereicherung des menschlichen Geistes, da Sprachen in ihrer Vielfalt verschiedene Weltansichten darstellen. Die entgegengesetzte universalistische Ansicht, die den Unterschieden in den Sprachen jegliche Bedeutung abspricht und, im Interesse ungehinderter Kommunikation, immer häufiger offen für eine einsprachige Welt eintritt (eine Englisch-sprachige Welt natürlich), macht alle jene Vorzüge der Mehrsprachigkeit unmöglich, die Yildiz befördern möchte. Exophone Literatur zeigt die Komplexität und Kontingenz unserer Welt auf. Das mag Einige beängstigen und einfache Wahrheiten schwieriger machen, aber diese Konsequenzen sind schlicht Anzeichen dafür, wie dringlich wir mehr über diese Komplexität und Kontingenz wissen müssen. 
Sprachabenteuer: Yoko Tawadas exophone Erkundungen des Deutschen

Deshalb braucht die Welt Kunst im Allgemeinen, zumindest wenn wir an einem Menschenbild mit Tiefendimension festhalten wollen.

Sprache, auch unsere Erstsprache, als ein Abenteuer zu erfahren, gibt uns die Frische der Sprache zurück und macht uns bewusst, wie sehr Sprache unsere Lebensbeziehungen bestimmt, folglich: wie sehr Sprache Leben ist. Friedrich Nietzsche, in „Ueber Wahrheit und Lüge im außermoralischen Sinne“, hatte die Erkenntnis, dass Sprache eine doppelte Metaphorisierung ist, was bedeutet, dass wir nur einen sehr vermittelten Zugang zur ,Realität' haben können. Aber wir vergessen diese Tatsache meistens und nehmen die Wörter à la lettre, als eine feststehende Wahrheit - für Nietzsche ist das eine lächerliche Illusion, Zeichen der größten Verlogenheit in der Menschheitsgeschichte (Nietzsche 2005: 873). Für Nietzsche führt das zu der Schlussfolgerung, dass die Welt nur ästhetisch gerechtfertigt werden kann. Diesen Gedanken der metaphorischen Bedingtheit von Sprache und damit von der Konstruiertheit unserer Welt weiterführend, behauptet der Vertreter des Russischen Formalismus Viktor Šklovskij in seinem Text „Auferweckung des Wortes“, dass wir die ursprünglich lebendigen Bilder der Worte, ihre Tropikalität, verloren haben, und dass wir Neuheit in die Sprache bringen müssen, um diese wieder zu spüren und um der Sprache ihre Kraft und Schönheit zurückzugeben. Ein Verfremdungselement erlaubt es, die Kraft des Wortes erneut zu spüren (Šklovskij 1973).

Tawada setzt der Sprachpolizei die Spielpolyglotte entgegen und demonstriert, wie, durch die Transgression der Grammatik, ein Dialog eröffnet wird:

Ich breche die Grammatik durch, den Stab der Sprachpolizisten. Ich durchbreche die Schlachtlinie und finde dort meine Feinde. Wie sehen sie aber aus? Ich muss sie erst erfinden. Ich finde sie er. Ich verstehe sie nicht. Ich stehe sie nicht ver. Ich spreche sie nicht an. Ich anspreche sie. (Tawada 2007: 28)

Ihr Spiel mit den Besonderheiten der deutschen Grammatik mit ihren trennbaren und nicht trennbaren Präfixen, die die Bedeutung der Wörter jeweils verändern, und mit Geschlecht, verweist sie auf die Merkwürdigkeiten und Implikationen solcher Grammatik und verwandelt sie in ein Spiel, um letztlich bei einer Ansprache des Anderen anzukommen. Im Folgenden sagt sie, wie sie „von einer großen Lust ergriffen (ist), die Buchstaben durcheinanderzubringen, um dem Spielplatz Wörterbuch seine poetische Ausstrahlung zurückzugeben“ (Tawada 2007: 37).

Kommen wir zum Abschluss nochmals auf das Abenteuer zurück. Die Geburt der ritterlichen Abenteuerideologie vom 11. bis zum 13. Jahrhundert lässt sich 
PAJEVIĆ

als eine der entscheidenden Wandlungen im menschlichen Selbstverständnis ansehen, da damit zum ersten Mal in der Menschheitsgeschichte das Vertraute systematisch preisgegeben wird, um das Fremde kennenzulernen. Neben dieser Überschreitung des kulturellen Horizontes wird auch das Zufällige der Existenz bejaht. Diese Haltung wird jetzt kultiviert als dem Menschen wesentlich und ihn auszeichnend. Darin ist die Möglichkeit des Wandels und der willentlichen Veränderung der Zustände bereits angelegt, also die Überwindung und Neugestaltung der Weltordnung, der Drang nach Neuem und Entwicklung, Fortschritt. Mit der Akzeptanz des Zufalls als konstitutivem Element ist das moderne Individuum geboren und der Gottesglaube untergraben (Sandkühler 1990). Jetzt ist der oder das Andere als konstitutives Element des Seins - und zwar als potentiell positives - anerkannt. Das ist eine Entwicklung, neben der laut Sandkühler Reformation und Französische Revolution zu bloßen Anekdoten der menschlichen Mentalitäts- und Sozialgeschichte verblassen.

Sprache als Abenteuer zu begreifen, ist also nichts Geringes. Eine andere Sprache zu erlernen wird in dieser Optik ein wesentliches Element des Menschseins. Es verweist ebenso auf die Möglichkeit einer Welt, in der ein Miteinander des Verschiedenen denkbar wird. Exophone Literatur weist uns den Weg. Grammatik als Abenteuer zu leben bedeutet, unsere geistigen Prozesse als Abenteuer zu leben, was wiederum bedeutet, uns selbst als Abenteuer zu leben. Es führt zu einem intensiven und bewussten Leben.

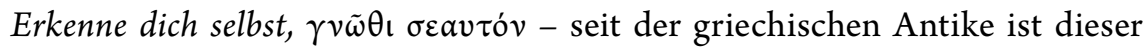
Imperativ wesentlicher Teil der Definition des Menschseins. Das ist wohl auch das, worauf Kafka sich in einem Brief vom 27. Januar 1904 an seinen Freund Oskar Pollock bezog, als er eine Literatur forderte, die die Axt darstellt, mit der das gefrorene Meer in uns aufgebrochen wird. Literatur sollte neue Perspektiven öffnen, uns die Welt anders denken lassen und derart ermöglichen, die Zustände in Frage zu stellen, und ebenso uns selbst. Das ist, was Sprachdenken, wie dasjenige Tawadas etwa, uns zwingt zu tun. Das ist in der Tat abenteuerlich. Abenteuer können gefährlich sein, sie können uns auf dem falschen Fu $\beta$ erwischen, aber solche Texte regen uns zum Denken an - und das ist in gewisser Weise, Immanuel Kant folgend, die Definition der Schönheit. ${ }^{6}$

\author{
Marko Pajević \\ marko.pajevic@ut.ee \\ University of Tartu \\ ESTONIA
}

\footnotetext{
Dieser Aufsatz ist eine leicht komplementierte Version eines auf Englisch erschienenen Artikels (Pajević 2019b).
} 
Sprachabenteuer: Yoko Tawadas exophone Erkundungen des Deutschen

\section{Literaturverzeichnis}

Buber, M. 1962 [1923]. Ich und Du. - Werke in drei Bänden. München/ Heidelberg: Kösel/Lambert Schneider, I: 77-170.

Cassin, B., ed., 2004. Vocabulaire européen des philosophies: Dictionnaire des intraduisibles. Paris: Seuil/Le Robert.

Dembeck, T., Uhrmacher, A. 2016. Vorwort: Erfahren oder erzeugt? Zum literarischen Leben der Sprachdifferenz. - T. Dembeck, A. Uhrmacher, eds., Das literarische Leben der Mehrsprachigkeit. Methodische Erkundungen. Heidelberg: Universitätsverlag C. Winter, 9-18.

Ette, O. 2012. Archipele der Literatur. Die neuzeitliche Tradition des Insulariums und das transarchipelische Schreiben Yoko Tawadas. - O. Gutjahr, ed., Yoko Tawada. Fremde Wasser. Vorlesungen und wissenschaftliche Beiträge. Tübingen: Konkursbuch Verlag Claudia Gehrke, 296-332.

Horkheimer, M. 1992 [1937]. Traditionelle und kritische Theorie. Frankfurt am Main: Fischer Wissenschaft.

Heimböckel, D. 2012. „Die Wörter dürfen nicht das sein, was sie meinen.“ Yoko Tawadas Beiträge zu einer interkulturellen Kritik der Sprache. - O. Gutjahr, ed., Yoko Tawada. Fremde Wasser. Vorlesungen und wissenschaftliche Beiträge. Tübingen: Konkursbuch Verlag Claudia Gehrke, 144-168.

Joseph, J. E. 2019. Introduction. - M. Pajević, ed., The Henri Meschonnic Reader. A Poetics of Society. Edinburgh: Edinburgh University Press, 1-14.

Leibniz, G. W. 1966 [1765]. Nouveaux Essais sur l'entendement humain. J. Brunschwig, ed. Paris: Garnier-Flammarion.

Nietzsche, F. 2005. Ueber Wahrheit und Lüge im außermoralischen Sinne. - Kritische Studienausgabe 1, G. Colli, M. Montinari, eds. München: dtv, 873-890.

Pajević, M. 2019. Glossary. - M. Pajević, ed., The Henri Meschonnic Reader. A Poetics of Society. Edinburgh: Edinburgh University Press.

Pajević, M. 2019b. Adventures in language. Yoko Tawada's explorations of German. Oxford German Studies, 48 (4), 494-504. https://doi.org/10.1080/00787191.201 9.1701874

Sandkühler, H. J., ed., 1990. Europäische Enzyklopädie zu Philosophie und Wissenschaften. Hamburg: Felix Meiner Verlag.

Schmitz-Evans, M. 2012. Yoko Tawadas Imaginationen zwischen westlichen und östlichen Schriftkonzepten und -metaphern. - O. Gutjahr, ed., Yoko Tawada. Fremde Wasser. Vorlesungen und wissenschaftliche Beiträge. Tübingen: Konkursbuch Verlag Claudia Gehrke, 269-295.

Šklovskij, V. 1972 [1914]. Die Auferweckung des Wortes. - W.-D. Stempel, ed., Texte russischer Formalisten II. München: Fink, 3-17.

Tawada, Y. 1998. Orpheus oder Izanagi/Till. Szenische Dichtung und Mythen und Märchen zwischen Europa und Asien. Tübingen: Konkursbuch Verlag.

Tawada, Y. 2001 [1998]. Verwandlungen. Tübinger Poetik-Vorlesung. Tübingen: Konkursbuch Verlag.

Tawada, Y. 2007. Sprachpolizei und Spielpolyglotte. Tübingen: Konkursbuch Verlag. 
PAJEVIĆ

Tawada, Y. 2010. Abenteuer der deutschen Grammatik. Tübingen: Konkursbuch Verlag. Tawada, Y. 2011 [1996]. Talisman. Tübingen: Konkursbuch Verlag.

Tawada, Y./Gutjahr, O. 2012. „In meinen Poetikvorlesungen werde ich viel über das Wasser sprechen, und der Tsunami kommt auch vor”. Yoko Tawada im Gespräch mit Ortrud Gutjahr. - O. Gutjahr, ed., Yoko Tawada. Fremde Wasser. Vorlesungen und wissenschaftliche Beiträge. Tübingen: Konkursbuch Verlag, 17-45.

Tawada, Y. 2016. Akzentfrei. Tübingen: Konkursbuch Verlag.

Tawada, Y. 2018 [2002]. Überseezungen. Tübingen: Konkursbuch Verlag.

Weissmann, D. 2016. Vom Sprechen mit zwei Mündern. Inszenierte Sprachverwirrung und präbabylonisches Fremdverstehen in Yoko Tawadas zweisprachigem Theaterstück Till. - T. Dembeck, A. Uhrmacher, eds., Das literarische Leben der Mehrsprachigkeit. Methodische Erkundungen. Heidelberg: Universitätsverlag C. Winter, 165-194.

Wertheimer, J. 2001 [1998]. Jürgen Wertheimer über Yoko Tawada. - Y. Tawada. Verwandlungen. Tübinger Poetik-Vorlesung. Tübingen: Konkursbuchverlag.

Yildiz, Y. 2012. Beyond the Mother Tongue. The Postmonolingual Condition. New York: Fordham University Press. 